Nota técnica 10 de AEMET

Rafael Ancell Trueba

Ramón Célis Díaz 
Aviso Legal: los contenidos de esta publicación podrán ser reutilizados,

citando la fuente y la fecha, en su caso, de la última actualización

\section{Edita:}

(C) Ministerio de Agricultura, Alimentación y Medio Ambiente

Agencia Estatal de Meteorología

Madrid, 2012

Catálogo de Publicaciones de la Administración General del Estado:

https://cpage.mpr.gob.es

NIPO: $281-12-014-0$

https://doi.org/10.31978/281-12-014-0

Agencia Estatal de Meteorología (AEMET)

C/ Leonardo Prieto Castro, 8

28040 Madrid

http://www.aemet.es/

@Aemet_Esp

https://www.facebook.com/AgenciaEstataldeMeteorologia 


\title{
TERMOPLUVIOMETRÍA DE CANTABRIA DURANTE EL PERIODO 1981-2010
}

\author{
Rafael Ancell Trueba(1) \\ Ramón Célis Díaz ${ }^{(2)}$ \\ (1) Unidad de Estudios y Desarrollos \\ (2) Sección de Climatología \\ Delegación Territorial en Cantabria
}

Nota Técnica No. 10

NIPO: 281-12-014-0

\section{Agencia Estatal de Meteorología (AEMET)}

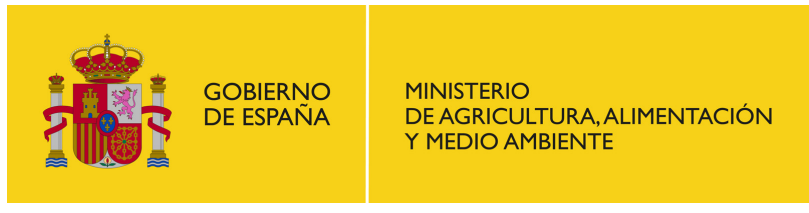

AEMet 


\section{$\underline{\text { Índice }}$}

1. Introducción

2. Datos utilizados.

3. Descripción general

4. Descripción estacional

5. Pluviometría

a. Régimen temporal

b. Distribución espacial

6. Termometría

a. Régimen temporal

b. Distribución espacial

7. Diagnóstico forense de la evolución reciente del Clima

8. Agradecimientos

9. Bibliografía 
1.

\section{INTRODUCCIÓN}

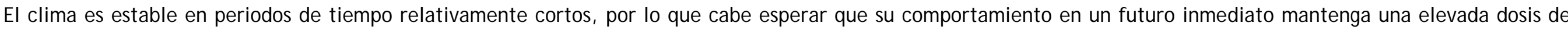

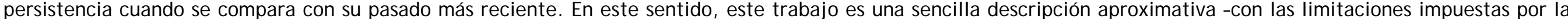

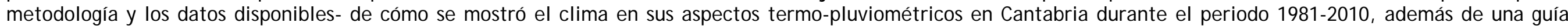

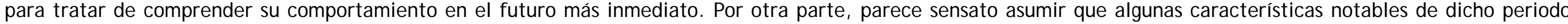
fueron singulares y probablemente no volverán a repetirse.

Toda la información relativa a la recopilación, depuración, homogeneización y relleno de lagunas en los datos, y para la obtención de las normales climatológicas del periodo 1981-2010, esta descrita en Cano \& Gutiérrez (2004) y Ancell \& Guillén (2013) y en sus referencias internas. También hay que añadir la valoración subjetiva que en algunos casos particulares ha sido determinante a la hora de seleccionar las estaciones más apropiadas en cada zona.

Finalmente, toda la información relativa a la metodología de interpolación para la realización de los mapas que se muestran en este trabajo, se puede consultar en Ancell \& Guillén (2013) y en sus referencias internas. Para ampliar conocimientos en el aspecto geográfico e histórico del Clima de Cantabria se recomienda consultar el excelente trabajo de Rodríguez J.J.(2011).

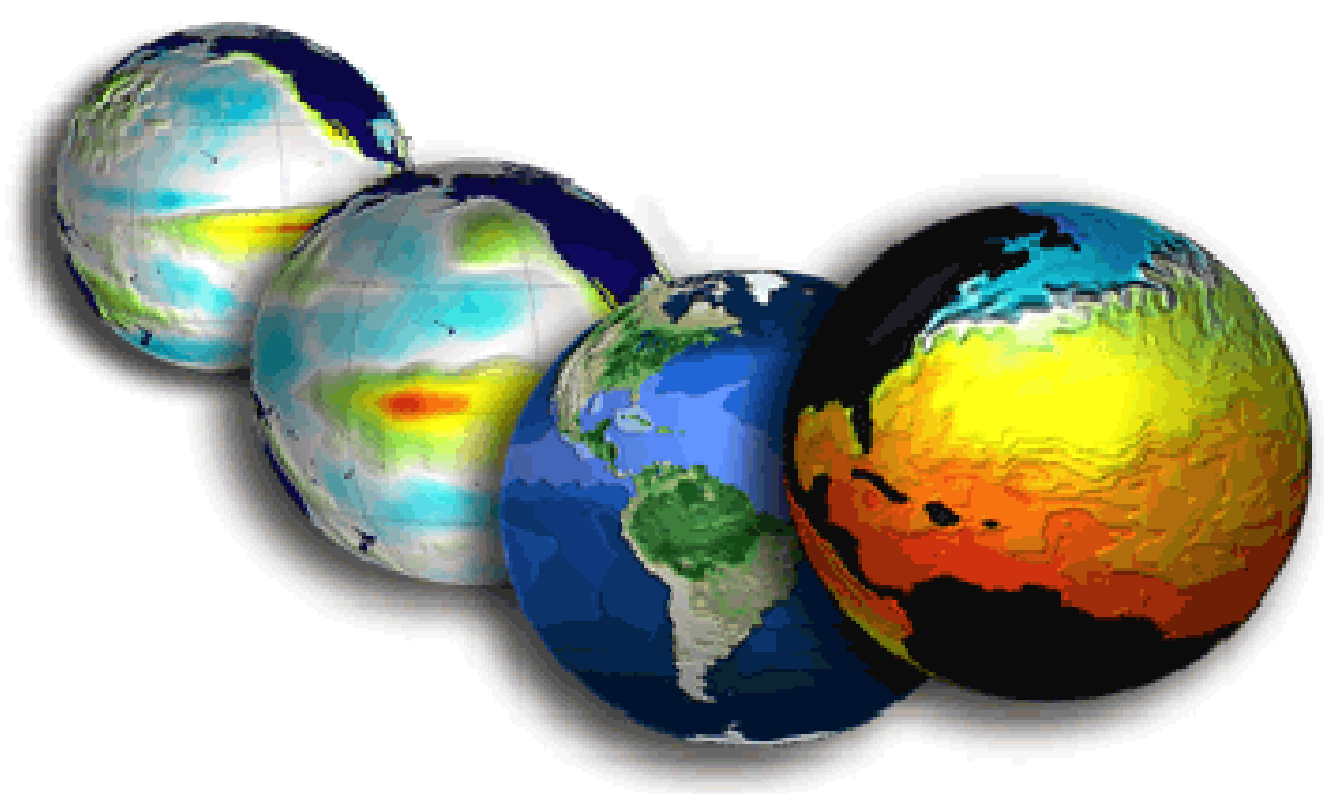




\section{DATOS UTILIZADOS}

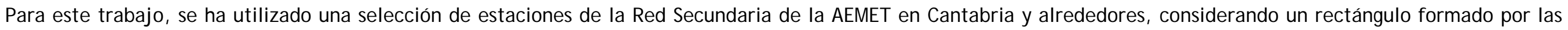

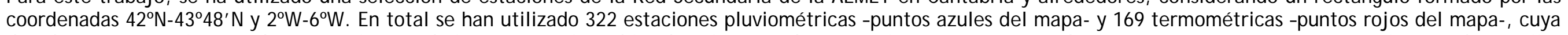

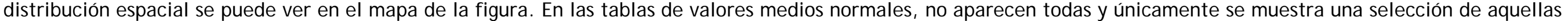
estaciones cuyo periodo real de funcionamiento se aj usta mejor al periodo 1981-2010, todos los detalles se pueden consultar en Ancell \& Guillén (2013).

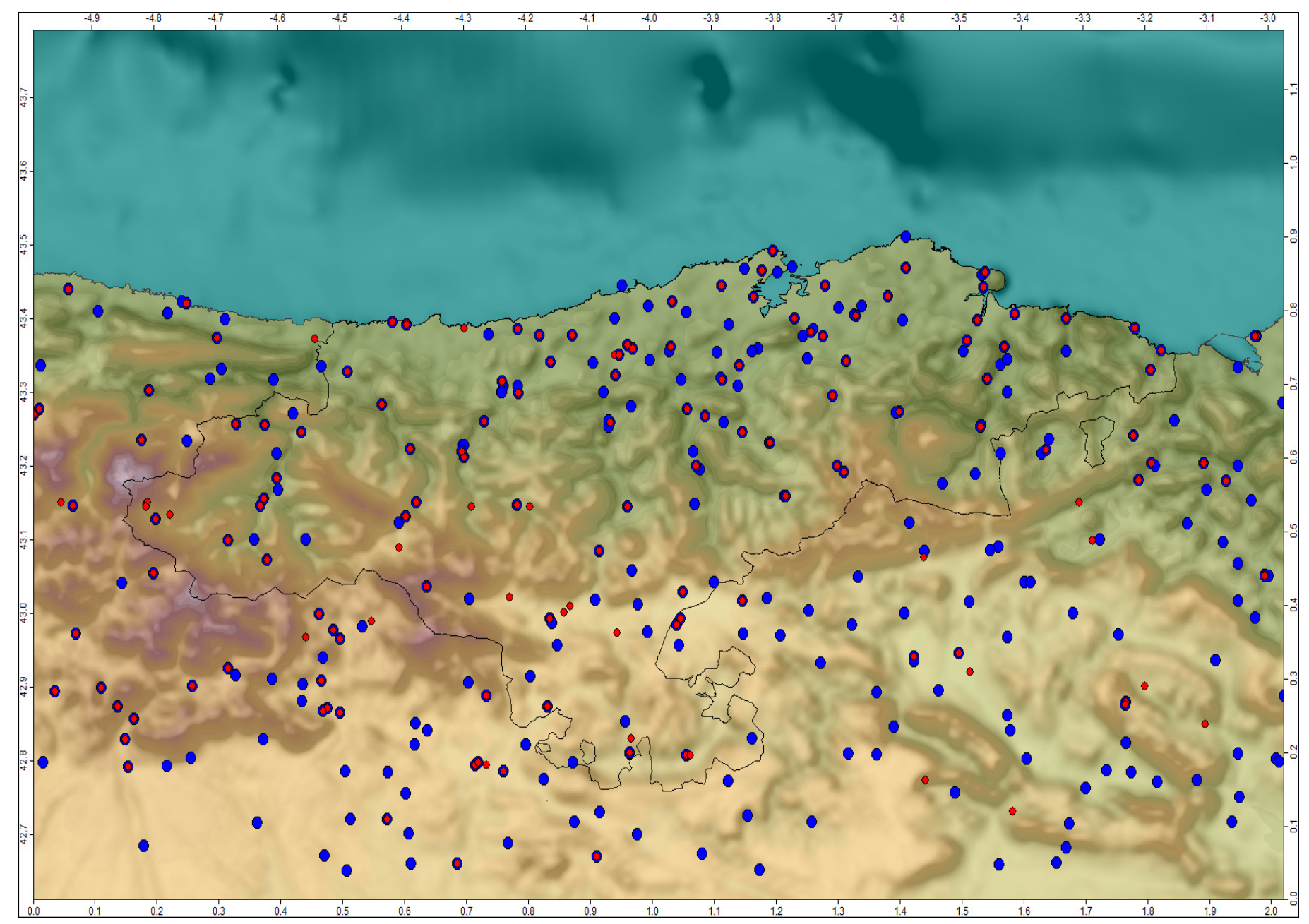


3.

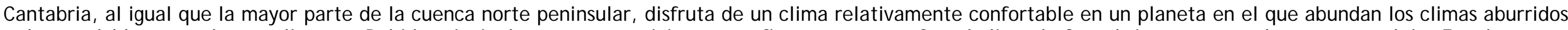

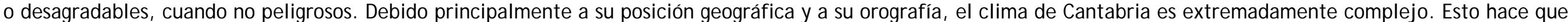

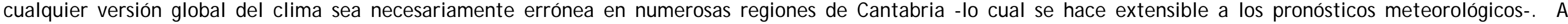

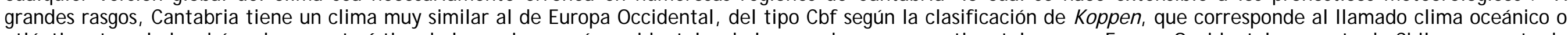

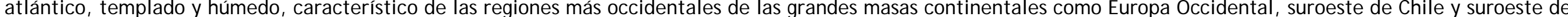

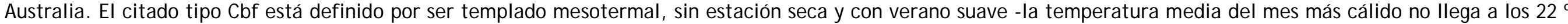

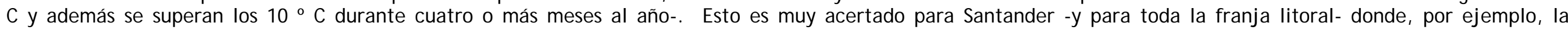

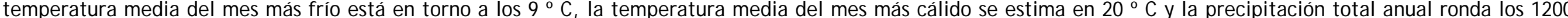

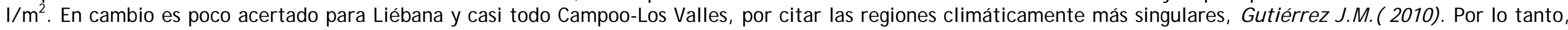
para ofrecer una perspectiva integral del clima de Cantabria, siendo realistas hemos de reconocer que es más lo que queda por descubrir que lo que sabemos.

4.

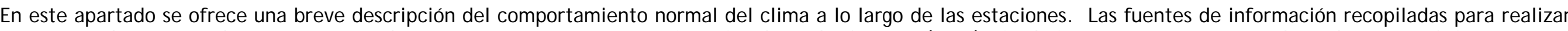

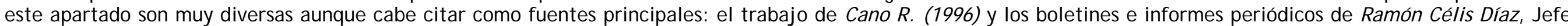

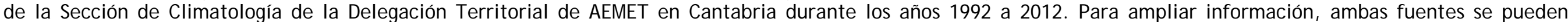
consultar en los archivos de la Delegación Territorial de AEMET en Cantabria.

\section{- $\quad$ EL INVIERNO EN CANTABRIA SUELE ALTERNAR ENTRE DOS TIPOS DE COMPORTAMIENTO}

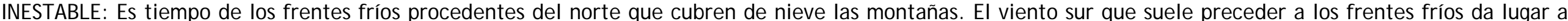

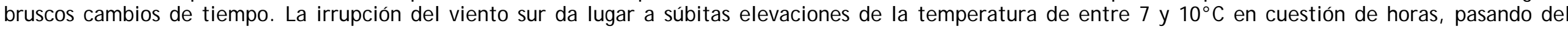
invierno al "verano" de un día para otro.

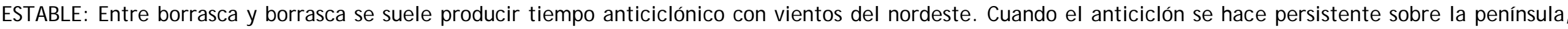
bloquea la llegada de frentes de lluvia, dando lugar a tiempo frío, seco y soleado que puede durar ente 15 y 45 días.

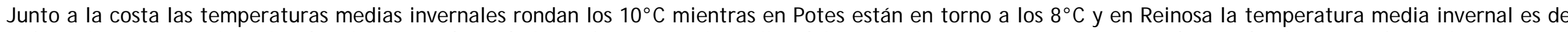

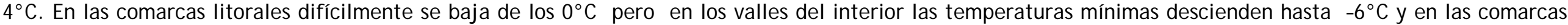
meridionales el termómetro puede bajar hasta los $-10^{\circ} \mathrm{C}$.

- $\quad$ LA PRIMAVERA EN CANTABRIA ES UN PERIODO DE CAMBIO CON GRANDES CONTRASTES (sol, Iluvia, frío y calor en el mismo día)

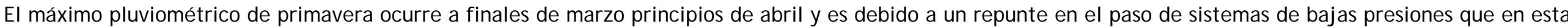

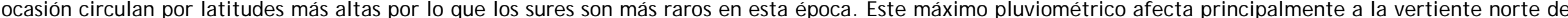

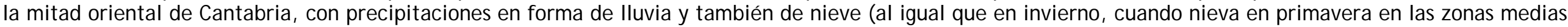

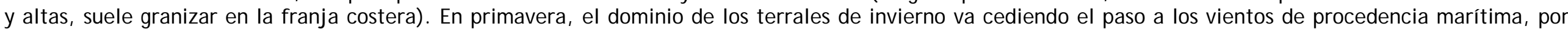

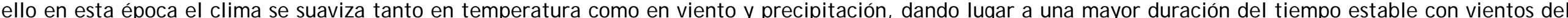

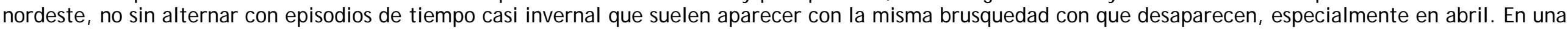

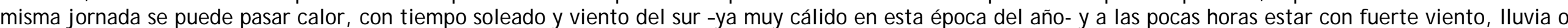




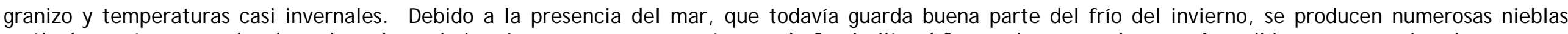

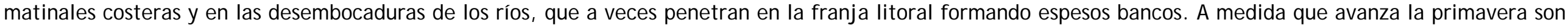
más frecuentes las tormentas, sobre todo en el interior. También al final de primavera son típicas las Iloviznas en el litoral oriental.

- $\quad$ EL VERANO EN CANTABRIA ES LA ÉPOCA MÁS APACIBLE Y AGRADABLE DEL AÑO (unos pocos días de Ilovizna y otros pocos días de agobio)

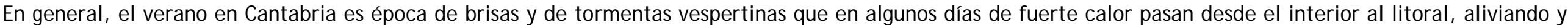

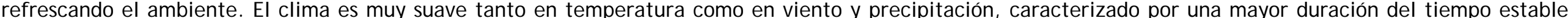

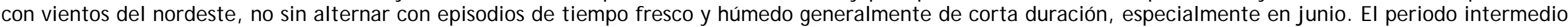

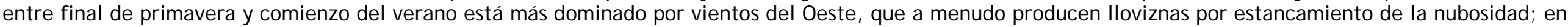

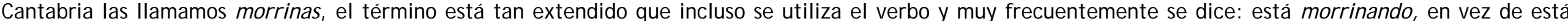

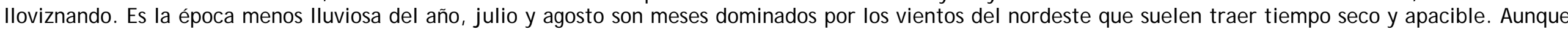

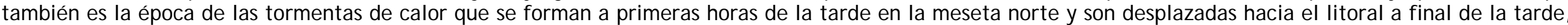

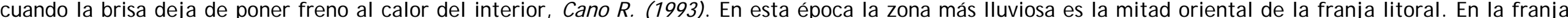
litoral es raro pasar de $30^{\circ} \mathrm{C}$ mientras que en los valles de la zona centro y sur, se pueden alcanzar los $36^{\circ} \mathrm{C}$.

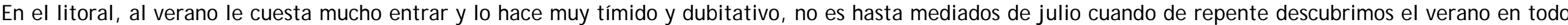

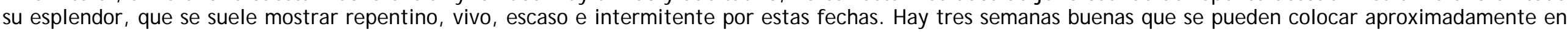

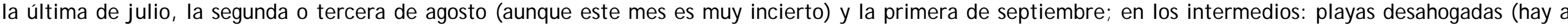
quien le gusta más así) y verano gastronómico de chubasquero, que tampoco esta nada mal.

- $\quad$ EL OTOÑO EN CANTABRIA ES ÉPOCA DE LLUVIASY DE VIENTOS DEL SUR (se alternan desordenadamente semanas Iluviosas con semanas secas)

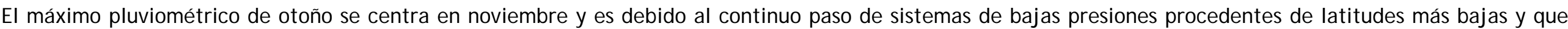

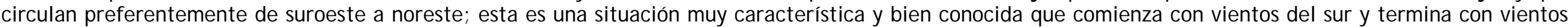

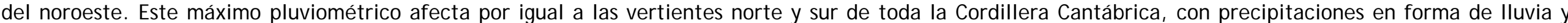

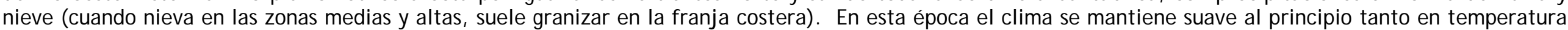

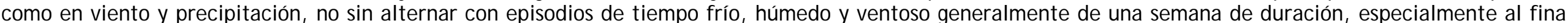

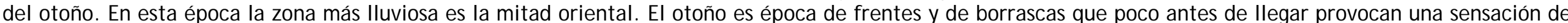

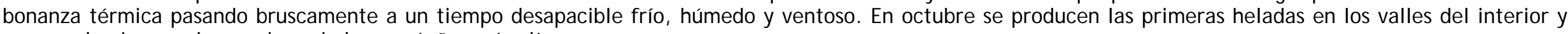
aparece la nieve en las cumbres de las montañas más altas. 
5.

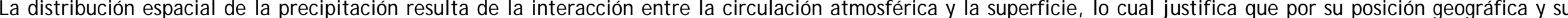

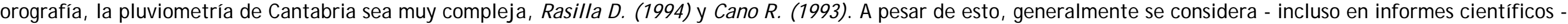

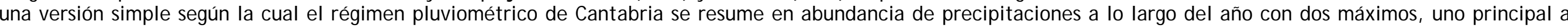

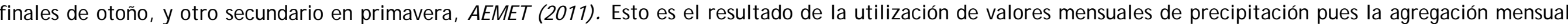
actúa como filtro para las oscilaciones de escalas de tiempo más pequeñas, Cano R. \& Gutiérrez J .M. (2004)

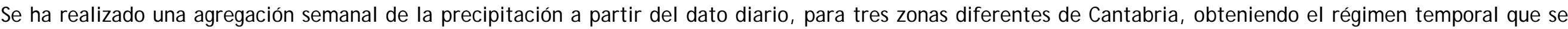
muestra en la figura.

Como se puede apreciar claramente en la figura, sin contradecir a modelo simple de la escala mensual, en el periodo 1981-2010 se produjeron oscilaciones muy significativas de escala intra-estacional. Aparecen 5 máximos, el absoluto de noviembre, el secundario de abril que muestra tener casi la mitad inmerso en marzo-, el tercero en Navidad, el cuarto entre septiembre y octubre y un quinto a final de febrero. Asimismo hay otros 5 mínimos, el principal, en verano, centrado entre julio y agosto, el secundario y bien conocido de mediados de marzo, un tercero a principio de febrero, el cuarto a finales de octubre y el quinto y muy significativo por encontrarse en plena época de Iluvia, centrado entre la segunda y la tercera semana de diciembre. El hecho de que estas oscilaciones aparezcan por igual en las tres zonas indica que son perturbaciones asociadas a grandes sistemas de presión que promediados durante el periodo 1981-2010 mostraron, como se ve en la figura, una significativa preferencia temporal; además, se ha constatado que estas oscilaciones afectaron a todo el dominio peninsular.

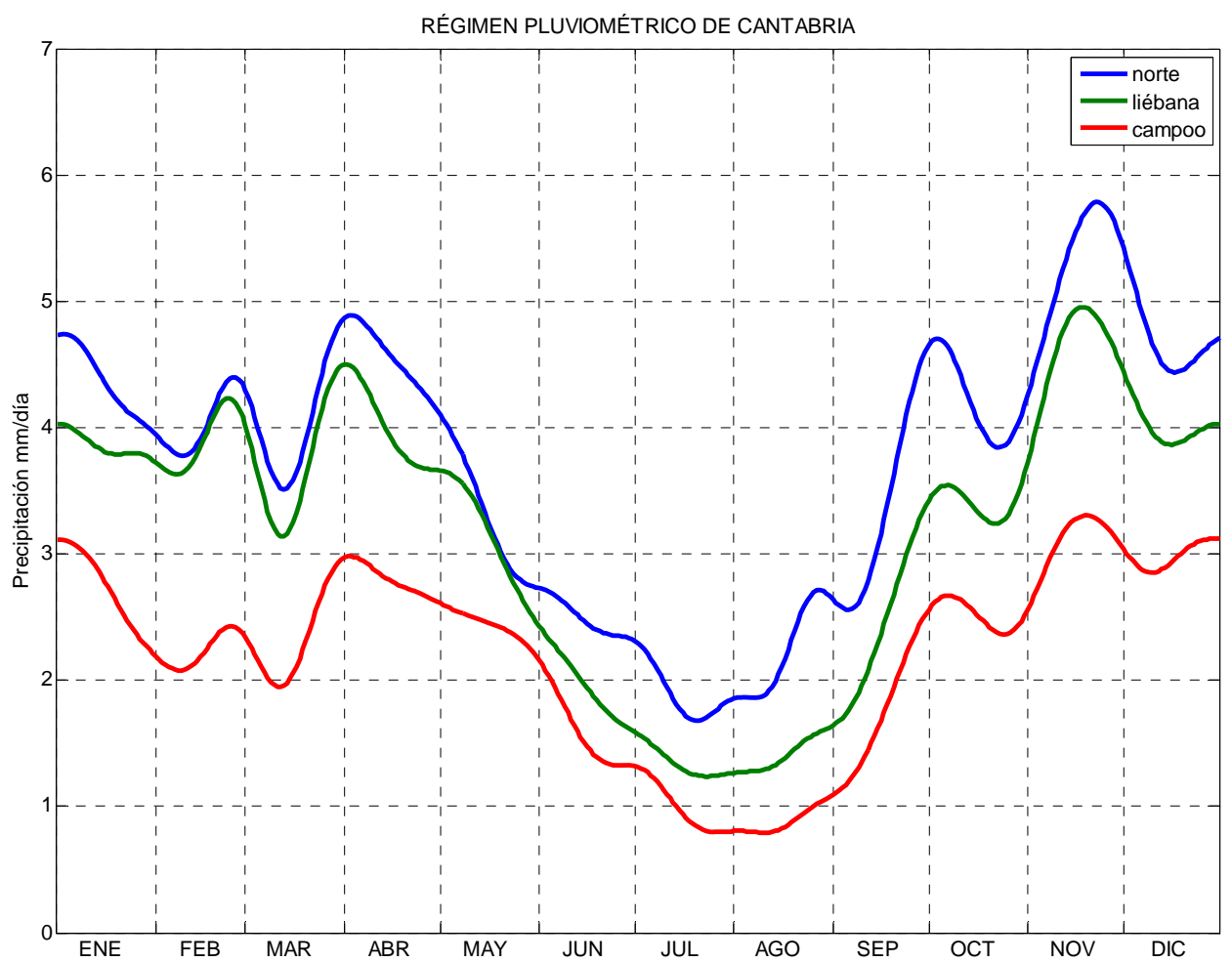




\section{b. Distribución espacial.}

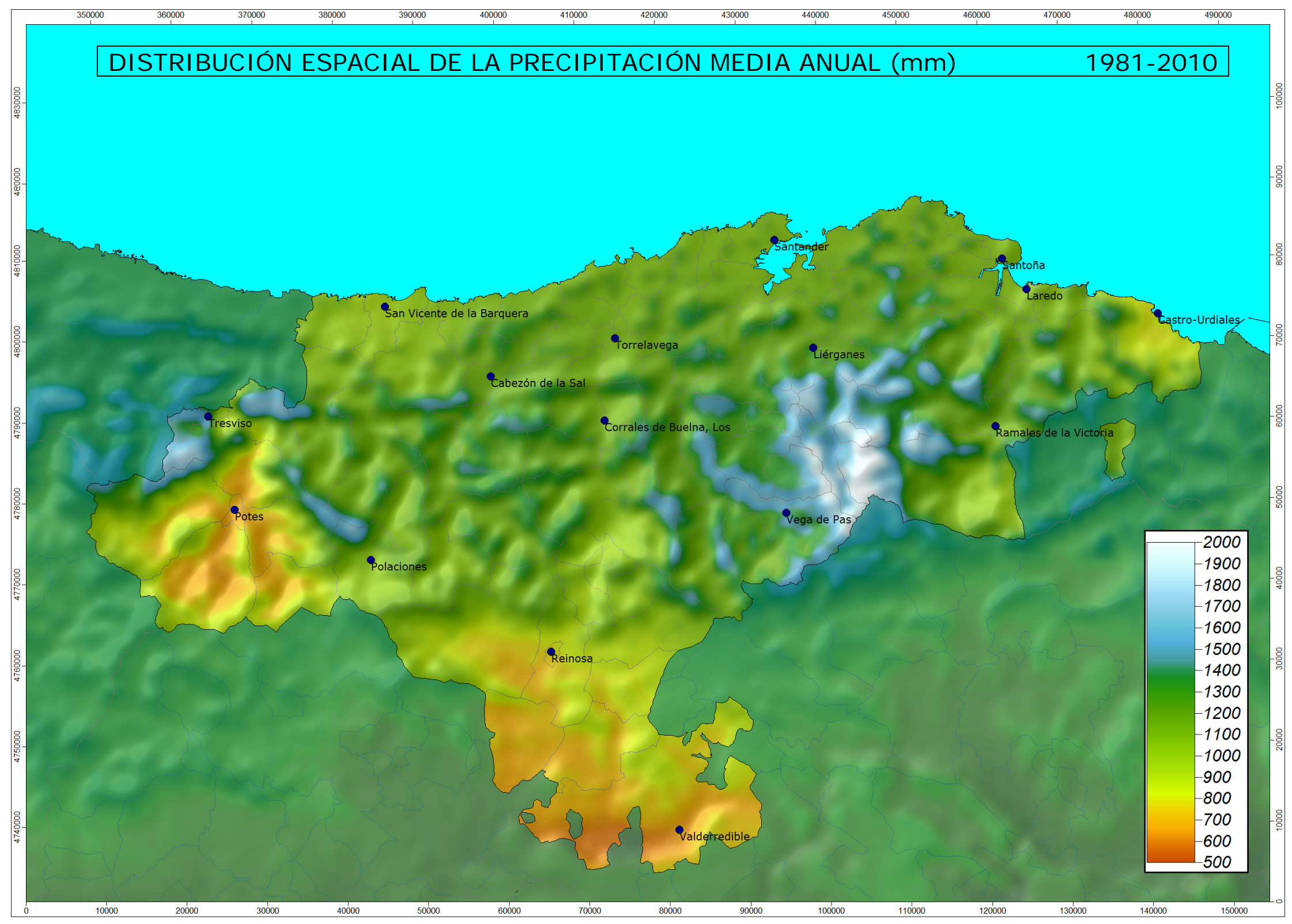



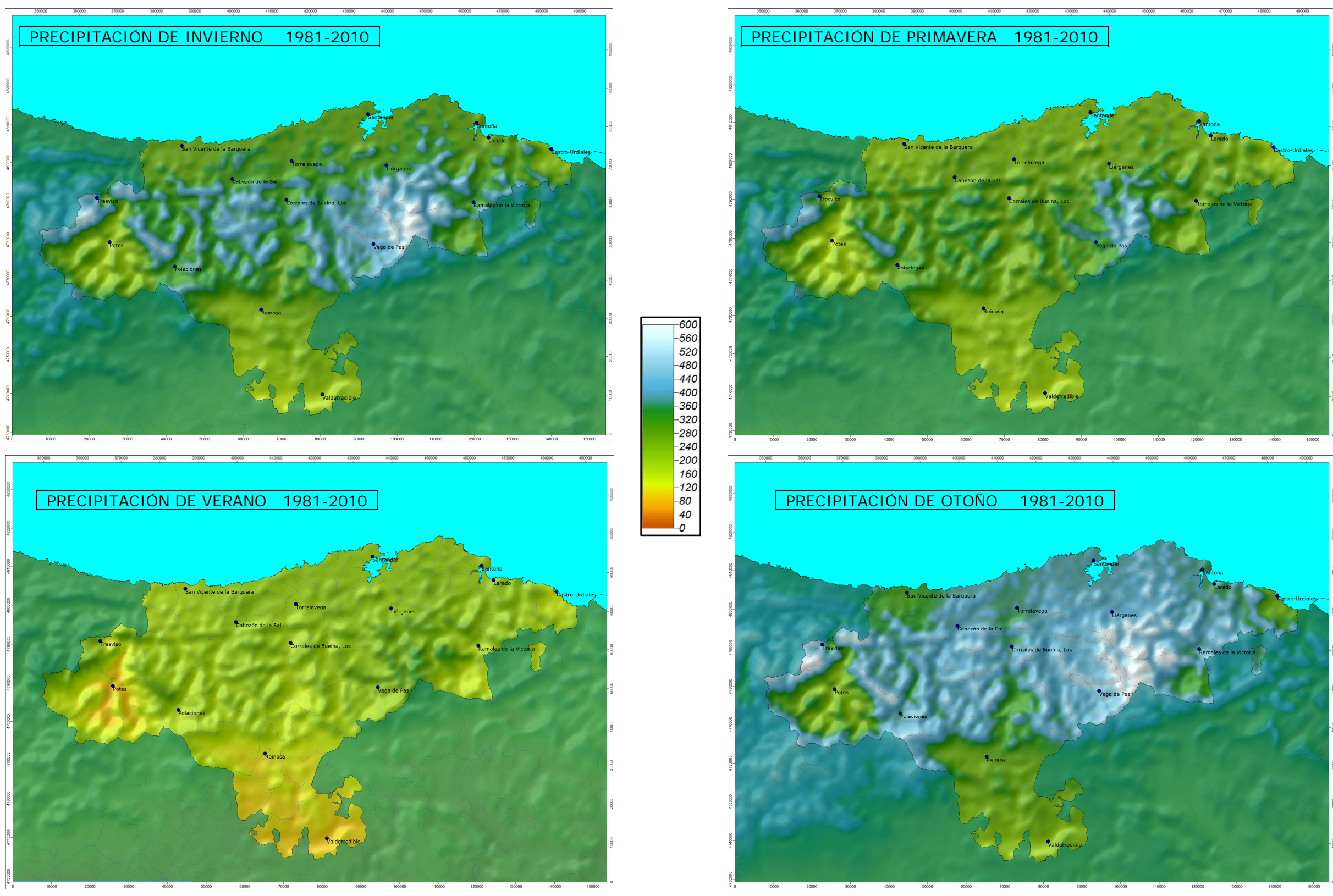
Las precipitaciones superan los $1000 \mathrm{~mm}$ año en el $80 \%$ de Cantabria.

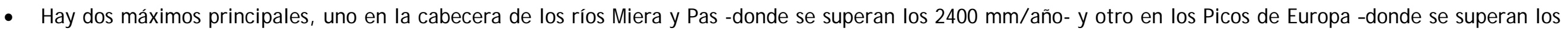
2000mm/ año-.

- Las zonas más secas se encuentran en el sur de Campoo-Los Valles y las cotas inferiores del Valle de Liébana -donde apenas se alcanzan los 700 mm/ año-.

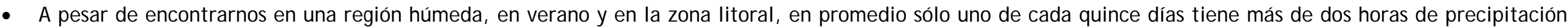
durante el día. 
Valores pluviométricos normales 1981-2010 en algunas estaciones de Cantabria, expresados en mm.

\begin{tabular}{|c|c|c|c|c|c|c|c|c|c|c|c|c|c|c|}
\hline I NDI CATI VO & ESTACIÓN & ENE & FEB & MAR & $\mathbf{A B R}$ & MAY & J UN & J UL & AGO & SEP & OCT & NOV & DIC & AÑo \\
\hline $1083 G$ & ONTON & 106 & 90 & 88 & 103 & 72 & 55 & 47 & 59 & 71 & 107 & 140 & 112 & 1051 \\
\hline $1084 \mathrm{~F}$ & GURI EZO & 157 & 128 & 129 & 148 & 103 & 69 & 56 & 77 & 93 & 150 & 193 & 160 & 1462 \\
\hline 1086 & LAREDO & 97 & 83 & 81 & 93 & 83 & 58 & 47 & 66 & 85 & 105 & 135 & 113 & 1046 \\
\hline 1087 & LIMPI AS & 126 & 107 & 106 & 122 & 87 & 63 & 51 & 73 & 89 & 132 & 158 & 131 & 1245 \\
\hline 1089A & ARREDONDO & 198 & 167 & 175 & 174 & 137 & 86 & 65 & 92 & 112 & 165 & 234 & 203 & 1809 \\
\hline 1090 & VI LLAR DE SOBA & 129 & 117 & 119 & 133 & 103 & 69 & 50 & 66 & 82 & 129 & 160 & 143 & 1301 \\
\hline 1090E & FRESNEDO DE SOBA & 109 & 88 & 98 & 108 & 87 & 55 & 42 & 54 & 59 & 90 & 126 & 110 & 1026 \\
\hline $1095 \mathrm{C}$ & UDALLA & 149 & 129 & 129 & 132 & 103 & 71 & 63 & 87 & 97 & 143 & 185 & 159 & 1446 \\
\hline 1095E & COTERI LLO DE AMPUERO & 142 & 125 & 121 & 126 & 99 & 69 & 56 & 83 & 92 & 138 & 179 & 146 & 1376 \\
\hline $1096 U$ & TRETO & 121 & 105 & 109 & 117 & 88 & 61 & 50 & 68 & 88 & 124 & 170 & 133 & 1235 \\
\hline 1098A & SANTOÑA & 106 & 89 & 86 & 99 & 78 & 56 & 48 & 69 & 82 & 116 & 142 & 113 & 1086 \\
\hline 1099 & HAZAS DE CESTO & 152 & 128 & 128 & 142 & 117 & 79 & 60 & 83 & 97 & 149 & 191 & 162 & 1487 \\
\hline $1099 \mathrm{U}$ & BAREYO & 134 & 109 & 109 & 125 & 93 & 70 & 59 & 86 & 102 & 146 & 188 & 150 & 1371 \\
\hline 1100 & CABO DE AJO (FARO) & 103 & 84 & 84 & 98 & 76 & 57 & 47 & 64 & 73 & 109 & 143 & 114 & 1051 \\
\hline 1100E & MERUELO & 148 & 117 & 120 & 142 & 106 & 80 & 68 & 95 & 109 & 162 & 202 & 157 & 1505 \\
\hline 11011 & SUESA & 120 & 96 & 93 & 110 & 84 & 63 & 56 & 80 & 85 & 126 & 164 & 130 & 1208 \\
\hline 1102A & LA CONCHA SAN ROQUE RI & 259 & 203 & 224 & 221 & 179 & 116 & 93 & 118 & 144 & 200 & 303 & 243 & 2304 \\
\hline 1104 & MI RONES & 198 & 165 & 171 & 186 & 139 & 93 & 81 & 106 & 123 & 174 & 237 & 199 & 1870 \\
\hline 11040 & EL MERCADI LLO DE LI ERGANES & 153 & 130 & 132 & 140 & 109 & 76 & 62 & 85 & 100 & 146 & 189 & 157 & 1480 \\
\hline 1105 & LA CAVADA (BARRIO DE ARRI BA) & 154 & 126 & 132 & 138 & 111 & 85 & 69 & 93 & 102 & 143 & 193 & 157 & 1503 \\
\hline $1105 \mathrm{U}$ & CECEÑAS & 136 & 112 & 112 & 130 & 103 & 70 & 59 & 84 & 95 & 136 & 181 & 139 & 1356 \\
\hline 11060 & SOLARES & 136 & 112 & 110 & 125 & 99 & 73 & 59 & 78 & 97 & 136 & 182 & 132 & 1339 \\
\hline 1107E & VI LLAVERDE DE PONTONES & 128 & 101 & 100 & 117 & 96 & 70 & 58 & 84 & 98 & 134 & 174 & 134 & 1293 \\
\hline 1109 & PARAYAS & 106 & 92 & 88 & 102 & 78 & 58 & 52 & 73 & 83 & 120 & 157 & 118 & 1129 \\
\hline 1110 & SANTANDER (ANTI GUO CENTRO) & 112 & 90 & 90 & 102 & 81 & 58 & 54 & 71 & 81 & 120 & 154 & 123 & 1135 \\
\hline 1110E & FARO LA MAGDALENA & 107 & 86 & 87 & 96 & 78 & 56 & 51 & 71 & 76 & 113 & 151 & 119 & 1092 \\
\hline 1111 & SANTANDER (CUETO) & 101 & 83 & 81 & 97 & 71 & 54 & 48 & 67 & 82 & 112 & 144 & 113 & 1053 \\
\hline 1112E & OJ AI Z & 112 & 91 & 91 & 105 & 78 & 61 & 53 & 76 & 86 & 119 & 160 & 122 & 1154 \\
\hline 1117B & VEGA DE PAS & 193 & 148 & 171 & 170 & 142 & 88 & 72 & 90 & 98 & 134 & 218 & 187 & 1711 \\
\hline 1120 & SEL DE LA CARRERA & 170 & 147 & 157 & 148 & 122 & 70 & 53 & 69 & 79 & 120 & 193 & 164 & 1494 \\
\hline 11210 & SAN MARTI N DE TORANZO & 139 & 120 & 123 & 123 & 99 & 65 & 47 & 63 & 74 & 111 & 170 & 140 & 1274 \\
\hline 11221 & ESCOBEDO DE VI LLAFUFRE & 147 & 122 & 140 & 137 & 112 & 73 & 60 & 79 & 92 & 126 & 193 & 150 & 1432 \\
\hline 1124E & VI LLACARRI EDO & 152 & 130 & 136 & 138 & 121 & 81 & 67 & 85 & 92 & 129 & 186 & 154 & 1471 \\
\hline 1127 & SAN MARTI N DE VI LLAFUFRE & 162 & 130 & 147 & 144 & 117 & 77 & 62 & 79 & 94 & 133 & 194 & 159 & 1499 \\
\hline $1127 \mathrm{U}$ & SANTA MARIA DE CAYON & 162 & 126 & 136 & 141 & 112 & 74 & 62 & 86 & 99 & 138 & 195 & 155 & 1487 \\
\hline 1128 & LA PENI LLA & 150 & 120 & 133 & 140 & 106 & 71 & 60 & 77 & 97 & 135 & 189 & 155 & 1434 \\
\hline 1129 & CASTAÑEDA & 149 & 116 & 126 & 136 & 107 & 73 & 58 & 80 & 92 & 136 & 193 & 143 & 1410 \\
\hline 1131 & VI OÑO & 135 & 105 & 110 & 121 & 97 & 71 & 60 & 84 & 92 & 134 & 175 & 137 & 1320 \\
\hline 11311 & TORRELAVEGA & 128 & 102 & 110 & 115 & 92 & 65 & 54 & 69 & 84 & 117 & 160 & 131 & 1227 \\
\hline
\end{tabular}




\begin{tabular}{|c|c|c|c|c|c|c|c|c|c|c|c|c|c|c|}
\hline I NDI CATI VO & ESTACIÓN & ENE & FEB & MAR & ABR & MAY & JUN & J UL & AGO & SEP & OCT & NOV & DIC & AÑO \\
\hline 1135 & BARCENA MAYOR & 138 & 115 & 121 & 121 & 107 & 63 & 49 & 60 & 66 & 114 & 164 & 134 & 1250 \\
\hline 1136E & TERAN & 139 & 119 & 120 & 121 & 108 & 66 & 52 & 60 & 71 & 105 & 159 & 134 & 1253 \\
\hline 1137R & RUENTE & 176 & 148 & 151 & 152 & 126 & 77 & 63 & 82 & 89 & 137 & 188 & 173 & 1562 \\
\hline 1139 & CABEZON DE LA SAL & 144 & 118 & 125 & 130 & 105 & 71 & 57 & 74 & 85 & 127 & 172 & 146 & 1354 \\
\hline 1139P & GOLBARDO & 143 & 119 & 124 & 127 & 96 & 68 & 54 & 75 & 90 & 124 & 170 & 144 & 1335 \\
\hline 1140E & PESQUERA & 111 & 95 & 95 & 104 & 97 & 55 & 48 & 52 & 63 & 89 & 132 & 113 & 1054 \\
\hline $1140 \mathrm{H}$ & ALDUESO & 111 & 99 & 95 & 106 & 94 & 59 & 46 & 50 & 63 & 98 & 129 & 111 & 1060 \\
\hline 1151B & CORRALES BUELNA & 135 & 114 & 124 & 120 & 96 & 61 & 45 & 60 & 72 & 112 & 166 & 142 & 1246 \\
\hline 1152 & MATA DE BUELNA & 148 & 113 & 127 & 134 & 104 & 66 & 52 & 70 & 87 & 129 & 191 & 151 & 1372 \\
\hline 1153E & VI ERNOLES & 126 & 105 & 111 & 119 & 90 & 70 & 54 & 73 & 87 & 118 & 158 & 126 & 1237 \\
\hline 11531 & REOCI N & 122 & 104 & 110 & 115 & 86 & 61 & 51 & 69 & 82 & 114 & 156 & 130 & 1199 \\
\hline 1155 & HI NOJ EDO & 109 & 94 & 95 & 104 & 82 & 59 & 50 & 64 & 76 & 106 & 139 & 117 & 1095 \\
\hline $1155 \mathrm{U}$ & NOVALES & 126 & 105 & 112 & 113 & 91 & 74 & 63 & 85 & 91 & 125 & 161 & 134 & 1279 \\
\hline 1156 & COBRECES & 120 & 96 & 104 & 108 & 88 & 69 & 64 & 82 & 84 & 116 & 155 & 123 & 1207 \\
\hline 1159E & SAN VI CENTE DE LA BARQUERA & 112 & 95 & 95 & 101 & 77 & 56 & 45 & 67 & 70 & 105 & 137 & 115 & 1074 \\
\hline 1160 & POLACI ONES (LA LAGUNA) & 134 & 117 & 122 & 130 & 123 & 68 & 60 & 67 & 78 & 115 & 167 & 144 & 1325 \\
\hline 11601 & EMBALSE DE LA COHI LLA & 124 & 110 & 115 & 124 & 120 & 66 & 55 & 65 & 72 & 105 & 154 & 131 & 1241 \\
\hline 11611 & ROZADIO & 118 & 99 & 102 & 109 & 100 & 59 & 44 & 59 & 66 & 101 & 144 & 118 & 1119 \\
\hline $1163 \mid$ & CELIS & 122 & 110 & 110 & 116 & 100 & 63 & 52 & 66 & 75 & 112 & 150 & 132 & 1208 \\
\hline 1165 & CAMI J ANES & 122 & 106 & 112 & 122 & 94 & 61 & 55 & 65 & 80 & 114 & 151 & 129 & 1211 \\
\hline 1165E & FUENTE DÉ-TELEFÉRI CO & 117 & 101 & 95 & 118 & 96 & 62 & 41 & 45 & 62 & 117 & 153 & 135 & 1142 \\
\hline 1167D & PIDO & 125 & 110 & 108 & 110 & 107 & 60 & 36 & 45 & 68 & 120 & 151 & 136 & 1175 \\
\hline 11700 & ENTERRIAS & 78 & 68 & 69 & 77 & 73 & 48 & 34 & 36 & 48 & 73 & 93 & 83 & 779 \\
\hline 1172E & POTES-LA TRESCORA & 65 & 57 & 58 & 63 & 58 & 34 & 24 & 27 & 39 & 61 & 79 & 64 & 629 \\
\hline 1173 & VALDEPRADO (PESAGUERO) & 92 & 77 & 70 & 87 & 81 & 48 & 32 & 32 & 50 & 78 & 110 & 95 & 853 \\
\hline $1174 I$ & TAMA & 74 & 65 & 63 & 69 & 60 & 35 & 25 & 28 & 39 & 62 & 97 & 74 & 690 \\
\hline 1175 & LA HERMI DA & 113 & 103 & 102 & 113 & 90 & 48 & 34 & 38 & 55 & 95 & 140 & 113 & 1044 \\
\hline 1175D & COLLADO LAS LLAVES-PEÑARRUBI A & 186 & 164 & 164 & 181 & 153 & 99 & 75 & 87 & 109 & 165 & 222 & 192 & 1797 \\
\hline $1175 I$ & PIÑERES & 151 & 135 & 129 & 144 & 120 & 71 & 48 & 62 & 82 & 134 & 189 & 154 & 1420 \\
\hline 1176 & TRESVISO & 201 & 186 & 185 & 195 & 157 & 88 & 64 & 76 & 99 & 162 & 254 & 203 & 1870 \\
\hline 1740 & ALTAMI RA & 133 & 103 & 111 & 123 & 92 & 77 & 66 & 89 & 95 & 135 & 173 & 142 & 1339 \\
\hline 2247 & MATAPORQUERA & 76 & 56 & 53 & 72 & 72 & 41 & 29 & 27 & 48 & 69 & 89 & 83 & 716 \\
\hline 9001D & NESTARES & 85 & 70 & 67 & 86 & 82 & 55 & 42 & 38 & 60 & 82 & 100 & 87 & 856 \\
\hline 9008E & VI LLASUSO & 109 & 87 & 97 & 95 & 88 & 45 & 33 & 38 & 51 & 88 & 128 & 114 & 972 \\
\hline 9013I & LA POBLACION DE YUSO & 141 & 116 & 113 & 107 & 97 & 49 & 39 & 38 & 56 & 94 & 160 & 131 & 1143 \\
\hline 9016 & CASTRI LLO DE VALDELOMAR & 58 & 44 & 41 & 61 & 63 & 42 & 27 & 24 & 45 & 60 & 70 & 66 & 603 \\
\hline $9016 x$ & CUBI LLO DE EBRO & 52 & 44 & 42 & 56 & 55 & 40 & 29 & 27 & 40 & 56 & 62 & 62 & 563 \\
\hline 9019 & POLI ENTES & 66 & 50 & 50 & 68 & 67 & 44 & 27 & 29 & 39 & 59 & 79 & 73 & 652 \\
\hline 9020E & VI LLAESCUSA DE EBRO & 65 & 55 & 53 & 64 & 61 & 34 & 23 & 26 & 33 & 60 & 84 & 77 & 633 \\
\hline
\end{tabular}




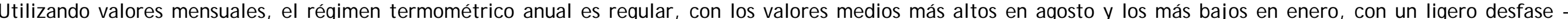

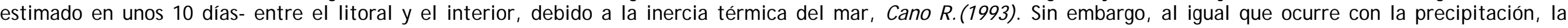

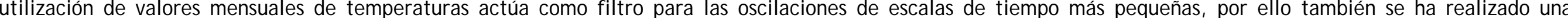

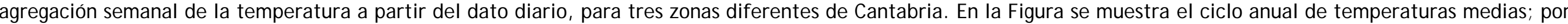
una parte se muestran las estaciones de la vertiente Norte (en azul) y por otra Liébana y Campoo en verde y rojo respectivamente.

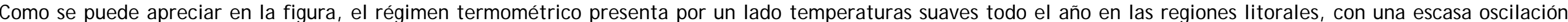

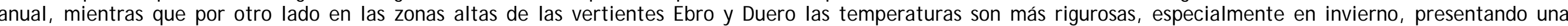

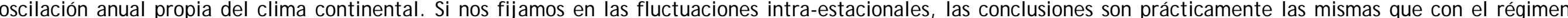

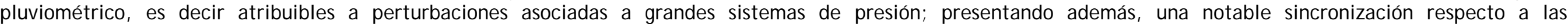
pluviométricas.

Si se observa la distribución espacial de temperaturas, los valles cántabros más fríos son Polaciones, Hermandad de Campoo de Suso, Campoo de Yuso, Enmedio, Valdeolea, Valdeprado del Río y Valderredible con temperaturas medias claramente inferiores a 10 o C y temperaturas mínimas absolutas anuales en torno a -15 o

Las zonas más templadas, aparte de toda la franj a litoral, son los valles del Besaya, Pas y Asón, así como el del Carranza entre

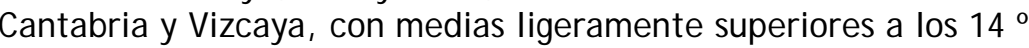
C y máximas absolutas anuales en torno a los 35 ㅇ C.

Como norma general, válida para toda la región, se observa un descenso térmico con la altura de unos 0.5 으 cada 100 metros. Este es un valor medio, que no se mantiene en el día a día; por ejemplo, en las mañanas anticiclónicas de los valles interiores, debido a que durante la noche el aire frío de las alturas se ha hundido al fondo de los valles por su mayor densidad, estos pueden ser tanto o más fríos que las cumbres, Cano R. (1993).

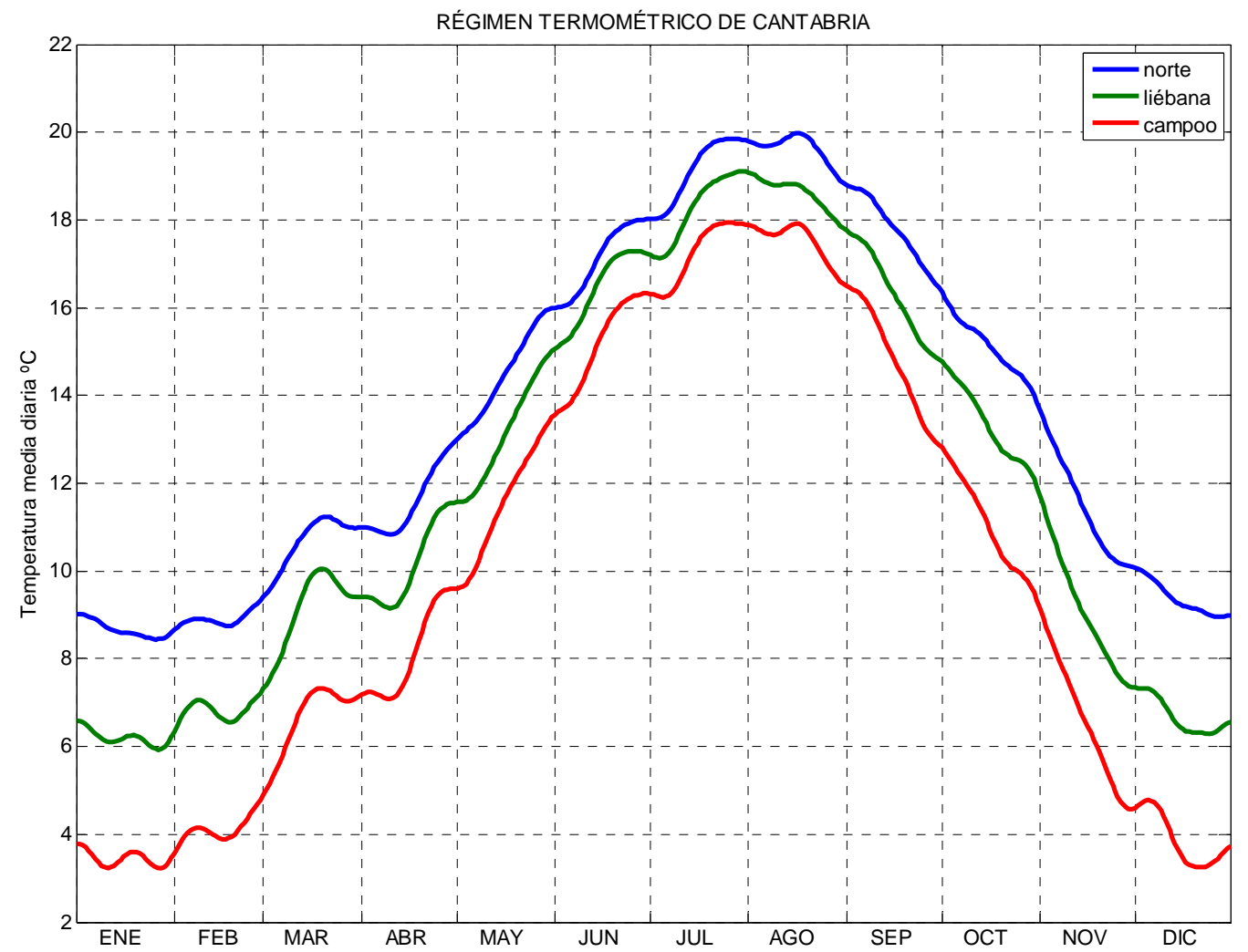

Página 13 de 20 


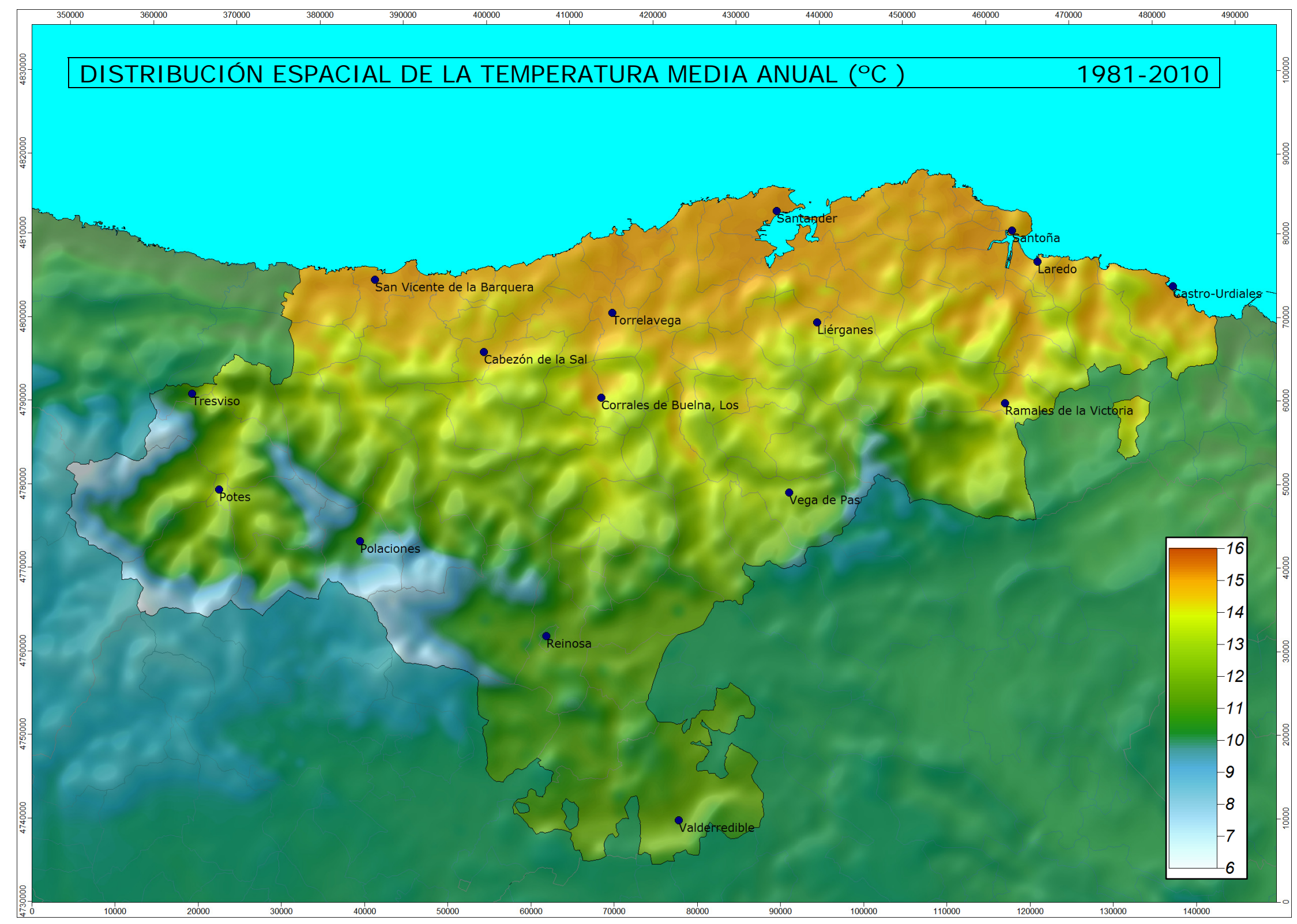



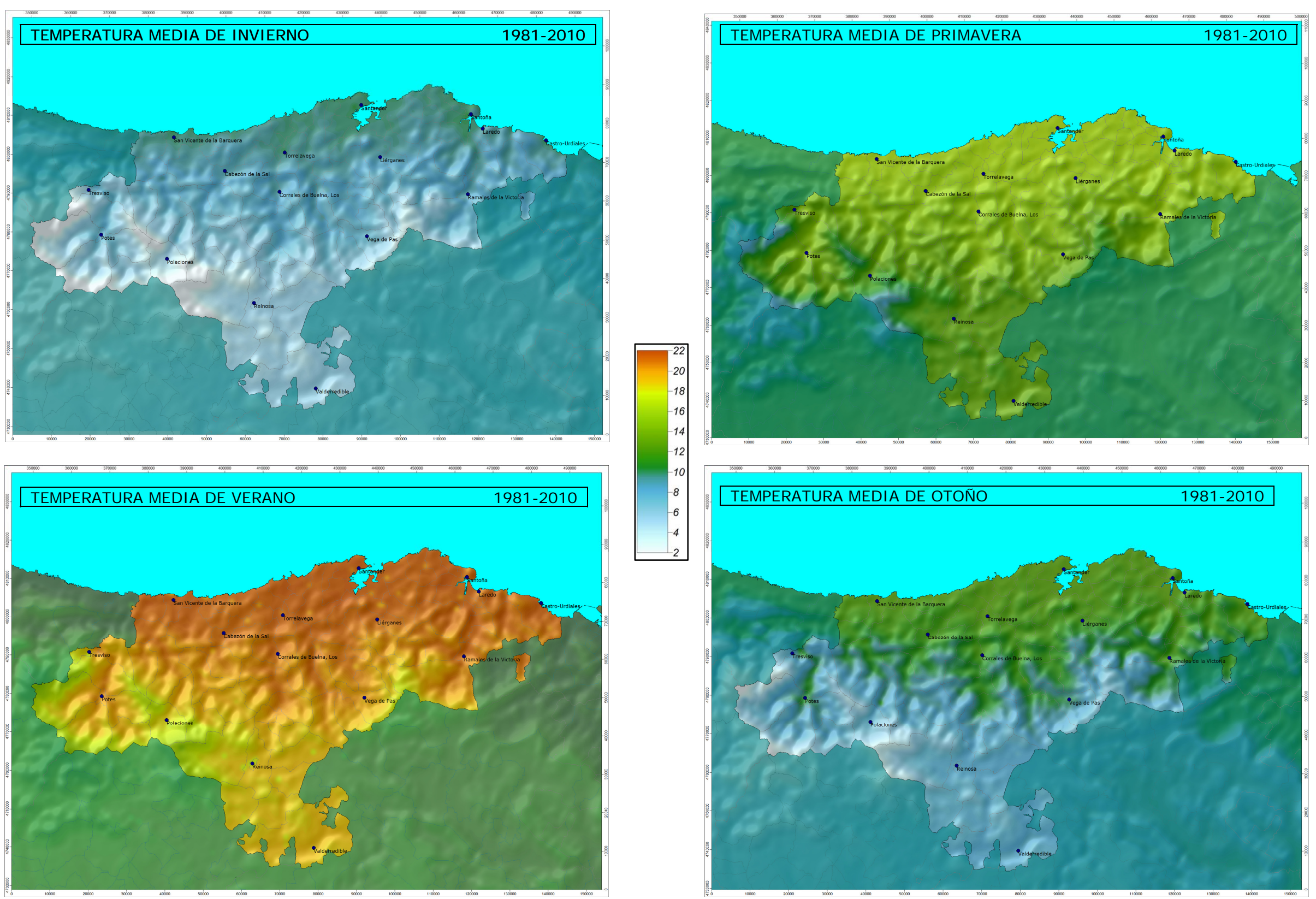
Valores termométricos normales 1981-2010 en al gunas estaciones de Cantabria, expresados en C.

\begin{tabular}{|c|c|c|c|c|c|c|c|c|c|c|c|c|c|c|}
\hline INDI CATIVO & ESTACI ÓN & ENE & FEB & MAR & ABR & MAY & JUN & J UL & AGO & SEP & OCT & NOV & DIC & AÑO \\
\hline $1083 X$ & ONTON & 10 & 10.1 & 11.7 & 12.5 & 14.9 & 17.5 & 19.4 & 20 & 18.4 & 16.2 & 12.6 & 10.7 & 14.5 \\
\hline 1089 & ARREDONDO & 7.7 & 8.3 & 10.3 & 11.4 & 14.6 & 17.4 & 19.1 & 19.4 & 17.7 & 14.9 & 10.8 & 8.4 & 13.3 \\
\hline $1095 \mathrm{C}$ & UDALLA & 9 & 9.3 & 11.2 & 12.1 & 15.1 & 17.7 & 19.7 & 20.1 & 18.3 & 15.6 & 11.8 & 9.6 & 14.1 \\
\hline $1096 U$ & TRETO & 9.3 & 9.4 & 11 & 12.1 & 15 & 17.5 & 19.5 & 19.8 & 18.2 & 15.7 & 12.1 & 10 & 14.1 \\
\hline 1098A & SANTOÑA & 9.9 & 10 & 11.6 & 12.5 & 15.1 & 17.7 & 19.6 & 20.1 & 18.6 & 16 & 12.5 & 10.6 & 14.5 \\
\hline $1099 U$ & BAREYO & 9.3 & 9.4 & 11 & 11.9 & 14.6 & 17.3 & 19.3 & 19.9 & 18.3 & 15.7 & 11.9 & 10 & 14 \\
\hline 1100E & MERUELO & 8.6 & 8.9 & 10.6 & 11.4 & 14.2 & 16.9 & 18.8 & 19.2 & 17.8 & 15.1 & 11.4 & 9.4 & 13.5 \\
\hline 1102A & LA CONCHA SAN ROQUE RIOMI ERA & 6.9 & 7.4 & 9.2 & 10.2 & 13.2 & 16.3 & 18.3 & 18.6 & 16.9 & 13.7 & 9.8 & 7.6 & 12.3 \\
\hline 1102D & SOBA-ALTO MI ERA & 6.4 & 6.6 & 8.5 & 9.3 & 12.6 & 15.1 & 17.1 & 17.6 & 16.1 & 13.3 & 9.4 & 7.3 & 11.6 \\
\hline 1104 & MI RONES & 8.6 & 9 & 10.8 & 11.9 & 14.8 & 17.4 & 19.2 & 19.5 & 17.9 & 15.1 & 11.3 & 9.3 & 13.7 \\
\hline $1105 U$ & CECEÑAS & 8.8 & 9 & 10.7 & 11.6 & 14.6 & 17.2 & 19.1 & 19.5 & 17.8 & 15.2 & 11.5 & 9.4 & 13.7 \\
\hline 1107B & HOZ DE ANERO-LA AGUERA & 8.8 & 9.1 & 10.7 & 11.8 & 14.7 & 17.3 & 19.4 & 19.8 & 18.1 & 15.5 & 11.5 & 9.4 & 13.8 \\
\hline 1109 & PARAYAS & 9.7 & 9.8 & 11.3 & 12.4 & 15.1 & 17.8 & 19.8 & 20.3 & 18.6 & 16.1 & 12.5 & 10.5 & 14.5 \\
\hline 1110 & SANTANDER (ANTI GUO CENTRO) & 10.3 & 10.4 & 11.8 & 12.7 & 15.3 & 17.8 & 19.8 & 20.4 & 18.8 & 16.4 & 12.9 & 10.9 & 14.8 \\
\hline 1111 & SANTANDER (CUETO) & 10.5 & 10.5 & 11.9 & 12.6 & 15.1 & 17.7 & 19.7 & 20.3 & 18.9 & 16.5 & 13.1 & 11.2 & 14.8 \\
\hline 1112E & OJAIZ & 9.4 & 9.5 & 11.1 & 12 & 14.7 & 17.4 & 19.3 & 19.9 & 18.2 & 15.7 & 12.1 & 10.1 & 14.1 \\
\hline 11220 & SOTO I RUZ & 9 & 9.2 & 11 & 12 & 14.8 & 17.4 & 19.2 & 19.8 & 18.1 & 15.5 & 11.8 & 9.7 & 14 \\
\hline 1124E & VI LLACARRI EDO & 8.1 & 8.4 & 10.2 & 11.2 & 14.3 & 17.1 & 19 & 19.4 & 17.6 & 15 & 11.1 & 8.8 & 13.3 \\
\hline 11311 & TORRELAVEGA & 9.5 & 9.7 & 11.3 & 12.4 & 15.1 & 17.8 & 19.7 & 20.1 & 18.6 & 16 & 12.2 & 10.2 & 14.4 \\
\hline 1135 & BARCENA MAYOR & 6.5 & 7.2 & 9.5 & 10.5 & 13.4 & 16.2 & 18.2 & 18.4 & 16.5 & 13.3 & 9.5 & 7.1 & 12.2 \\
\hline $1136 E$ & TERAN & 7.9 & 8.3 & 10.2 & 11.2 & 14.1 & 17 & 18.9 & 19.3 & 17.7 & 14.7 & 10.7 & 8.6 & 13.2 \\
\hline 1137R & RUENTE & 7.4 & 7.8 & 9.8 & 11 & 14 & 16.7 & 18.8 & 19.1 & 17.2 & 14.3 & 10.3 & 8.1 & 12.9 \\
\hline 1139D & CABEZON DE LA SAL LA PLATA & 9.6 & 9.8 & 11.3 & 12.1 & 14.6 & 17.1 & 18.9 & 19.4 & 18.1 & 15.7 & 12.2 & 10.3 & 14.1 \\
\hline 1139P & GOLBARDO & 8.3 & 8.8 & 10.4 & 11.5 & 14.4 & 17.1 & 19 & 19.4 & 17.7 & 15 & 11.1 & 8.9 & 13.5 \\
\hline 1140E & PESQUERA & 5.7 & 6.4 & 8.6 & 9.9 & 13.2 & 16.3 & 18.2 & 18.4 & 16.4 & 13.2 & 8.8 & 6.4 & 11.8 \\
\hline 1151 & LOS CORRALES DE BUELNA & 8.5 & 8.9 & 10.4 & 11.3 & 14.2 & 17 & 19.1 & 19.2 & 17.6 & 14.9 & 11.1 & 9.1 & 13.4 \\
\hline 1159E & SAN VI CENTE & 9.1 & 9.3 & 10.9 & 11.7 & 14.2 & 16.9 & 19.1 & 19.7 & 17.8 & 15.6 & 12.1 & 10 & 13.9 \\
\hline 1167D & PIDO & 4 & 4.9 & 7 & 8.2 & 11.4 & 14.8 & 16.9 & 17.1 & 14.7 & 11.2 & 7 & 4.6 & 10.1 \\
\hline 11700 & ENTERRIAS & 6.1 & 6.8 & 9 & 10 & 13 & 16.5 & 18.6 & 19 & 17.2 & 13.6 & 9.3 & 6.9 & 12.2 \\
\hline $1172 E$ & POTES-LA TRESCORA & 6.6 & 7.6 & 10 & 11.2 & 14.6 & 17.9 & 20.1 & 20.4 & 18.1 & 14.7 & 10 & 7.2 & 13.2 \\
\hline 1174 & TAMA & 6.9 & 7.8 & 10.2 & 11.4 & 14.6 & 17.9 & 19.9 & 20.1 & 18 & 14.6 & 10.2 & 7.5 & 13.3 \\
\hline $1175 I$ & PIÑERES & 6.3 & 6.6 & 8.3 & 9.3 & 12.1 & 14.9 & 16.6 & 17.1 & 15.7 & 13 & 9.1 & 7 & 11.3 \\
\hline 1176 & TRESVISO & 5.2 & 5.6 & 7.5 & 8.2 & 11.1 & 14.1 & 16 & 16.4 & 15 & 12.1 & 8.2 & 6.2 & 10.5 \\
\hline 2247 & MATAPORQUERA & 2.9 & 4.1 & 6.7 & 8.2 & 11.6 & 15.5 & 18.2 & 18.4 & 15.2 & 11 & 6.6 & 4 & 10.2 \\
\hline 9001D & REI NOSA-NESTARES & 2.7 & 3.4 & 5.6 & 7.1 & 10.5 & 13.7 & 16 & 16.2 & 13.6 & 10.2 & 5.8 & 3.4 & 9 \\
\hline 9008 & ARROYO DE VALDEAR & 3.7 & 4.3 & 6.5 & 7.8 & 11.2 & 14.4 & 16.6 & 16.9 & 14.5 & 11.2 & 6.8 & 4.6 & 9.9 \\
\hline 90110 & LA POBLACI ON DE YUSO I BERD & 2.3 & 3.3 & 6.3 & 7.5 & 10.8 & 15 & 17 & 17 & 14.5 & 10.2 & 6.2 & 3.1 & 9.4 \\
\hline $9015 \mathrm{C}$ & OTERO DEL MONTE & 2.6 & 3.5 & 6.1 & 7.4 & 11 & 14.6 & 17.2 & 17.5 & 14.7 & 10.7 & 6.1 & 3.6 & 9.6 \\
\hline 9016X & CUBI LLO DE EBRO & 3.5 & 4.4 & 6.9 & 8.4 & 12.1 & 15.4 & 18.2 & 18.2 & 15.3 & 11.6 & 6.7 & 4.2 & 10.4 \\
\hline 9019B & POLIENTES-CASYC & 3.6 & 4.6 & 7.1 & 8.5 & 12 & 15.7 & 18.3 & 18.4 & 15.2 & 11.2 & 6.8 & 4 & 10.5 \\
\hline
\end{tabular}


7.

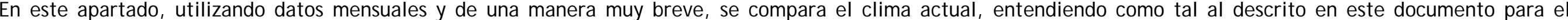

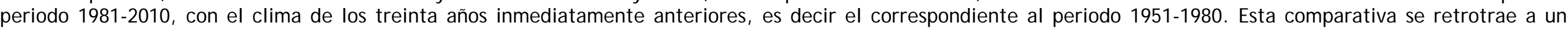

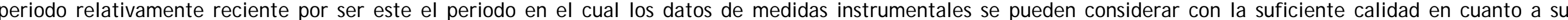

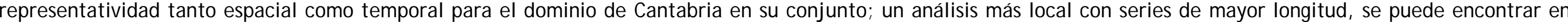
Rodríguez J .J . (2011)

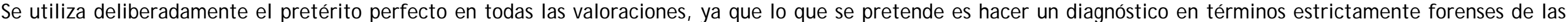

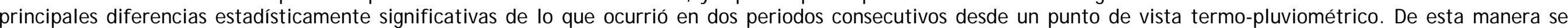

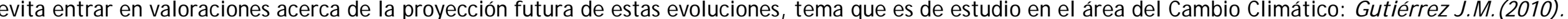

- En el periodo 1951-1980 la temperatura media anual de Cantabria en su conj unto fue de $12.42^{\circ} \mathrm{C}$, mientras que en el periodo 1981-2010 el promedio fue de 12.870C, es decir 0.45C más cálido. Esta diferencia fue más notable en los meses de junio, agosto y noviembre con un incremento ligeramente superior a $0.6 \circ \mathrm{C}$, mientras que en septiembre y febrero apenas alcanzó los $0.2 \circ$ ․․ En cuanto a la distribución geográfica de las diferencias, fue muy homogénea al igual que ocurrió con la precipitación.

- En el periodo 1951-1980 la precipitación media anual de Cantabria en su conj unto fue de $1333 \mathrm{~mm}$, mientras que en el periodo 1981-2010 el promedio fue de 1209mm: un 10\% menos. Este descenso fue más notable en los meses de junio, octubre y diciembre casi con un $20 \%$ menos, el resto de los meses fueron prácticamente iguales, si acaso con un leve déficit medio del $5 \%$ En cuanto a la distribución geográfica de las diferencias, fue muy homogénea.

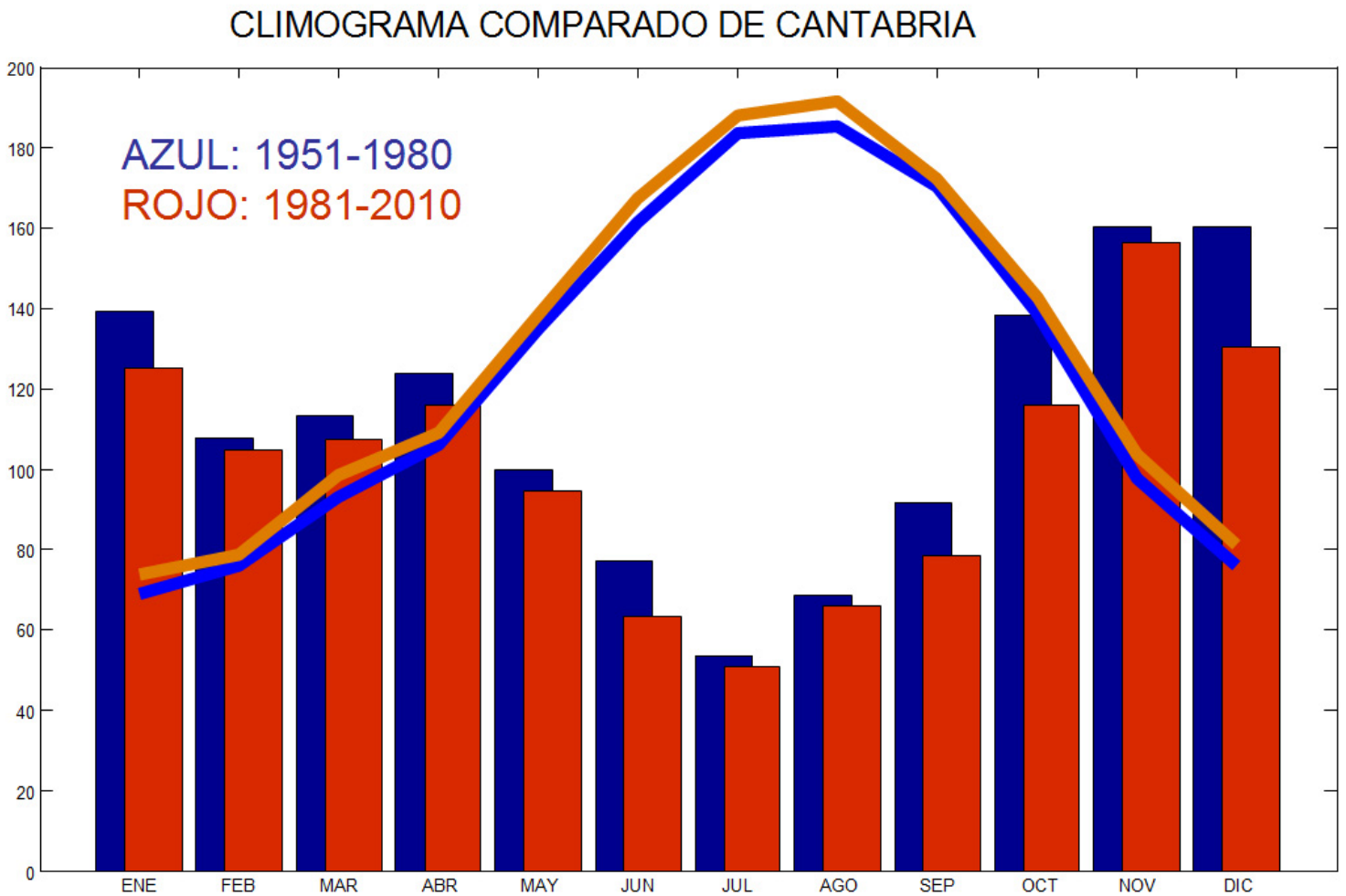


PRECIPITACIÓN

\begin{tabular}{|c|c|c|c|c|c|c|c|}
\hline 1951-1980 & $\mathrm{mm} /$ año & 1981-2010 & $\mathrm{mm}$ año & 1951-1980 & ${ }^{\circ} \mathrm{C}$ & $1981-2010$ & $\stackrel{\circ}{\circ}$ \\
\hline 1957 & 1067 & 1989 & 848 & 1956 & 11.4 & 1984 & 12.2 \\
\hline 1955 & 1097 & 2006 & 942 & 1972 & 11.5 & 1986 & 12.2 \\
\hline 961 & 1122 & 2001 & 995 & 1954 & 12 & 1993 & 12.2 \\
\hline 1970 & 1139 & 1995 & 1059 & 1963 & 12.1 & 1991 & 12.3 \\
\hline 1964 & 1140 & 1997 & 1066 & 1971 & 12.1 & 2010 & 12.3 \\
\hline 1968 & 1174 & 1994 & 1083 & 1973 & 12.1 & 1992 & 12.4 \\
\hline 1976 & 1182 & 1987 & 1132 & 1974 & 12.1 & 2007 & 12.5 \\
\hline 1956 & 1221 & 1981 & 1136 & 1980 & 12.2 & 1996 & 12.6 \\
\hline 1953 & 1239 & 2000 & 1138 & 1951 & 12.3 & 2005 & 12.6 \\
\hline 1962 & 1242 & 1988 & 1150 & 1969 & 12.3 & 2008 & 12.6 \\
\hline 1963 & 1279 & 2003 & 1151 & 1970 & 12.3 & 1985 & 12.7 \\
\hline 1977 & 1300 & 1985 & 1165 & 1975 & 12.3 & 2004 & 12.7 \\
\hline 1958 & 1301 & 1983 & 1176 & 1976 & 12.3 & 1981 & 12.8 \\
\hline 1967 & 1301 & 1990 & 1186 & 1978 & 12.3 & 2001 & 12.8 \\
\hline 1972 & 1331 & 2002 & 1194 & 1979 & 12.3 & 1988 & 12.9 \\
\hline 1951 & 1338 & 1991 & 1198 & 1962 & 12.4 & 1999 & 12.9 \\
\hline 1965 & 1347 & 1993 & 1198 & 1965 & 12.4 & 2009 & 12.9 \\
\hline 1969 & 1350 & 2009 & 1199 & 1977 & 12.4 & 1994 & 13 \\
\hline 1954 & 1361 & 1999 & 1210 & 1957 & 12.5 & 1998 & 13 \\
\hline 1973 & 1401 & 2007 & 1217 & 1967 & 12.5 & 2000 & 13 \\
\hline 1980 & 1416 & 2005 & 1264 & 1953 & 12.6 & 1982 & 13.1 \\
\hline 1959 & 1444 & 1982 & 1265 & 1960 & 12.6 & 1983 & 13.1 \\
\hline 1966 & 1449 & 1998 & 1280 & 1952 & 12.7 & 1987 & 13.1 \\
\hline 1974 & 1464 & 2004 & 1314 & 1958 & 12.8 & 2002 & 13.1 \\
\hline 1971 & 1478 & 1986 & 1317 & 1964 & 12.8 & 1990 & 13.3 \\
\hline 1960 & 1483 & 2010 & 1431 & 1966 & 12.9 & 1995 & 13.3 \\
\hline 1952 & 1518 & 1984 & 1434 & 1968 & 12.9 & 2006 & 13.5 \\
\hline 1978 & 1535 & 1996 & 1442 & 1955 & 13 & 2003 & 13.6 \\
\hline 1975 & 1570 & 1992 & 1491 & 1959 & 13.1 & 1989 & 13.8 \\
\hline 1979 & 1721 & 2008 & 1594 & 1961 & 13.6 & 1997 & 13.8 \\
\hline MEDIA & 1333 & MEDIA & 1209 & MEDIA & 12.42 & MEDIA & 12.87 \\
\hline
\end{tabular}

Tabla con las series ordenadas de menor a mayor
TEMPERATURA

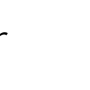




\section{PRECIPITACIÓN}

En la serie 1951-1980 (columnas azul pálido) el año más seco fue 1957 con 1067 mm y el más lluvioso 1979 con 1721 mm.

En la serie 1981-2010 (columnas azul oscuro) el año más seco fue 1989 con 848 mm y el más lluvioso 2008 con 1594 mm.

\section{TEMPERATURA}

En la serie 1951-1980 (columnas amarillo pálido) el año más cálido fue 1961 con 13.6 ㄷ C y el más frío 1956 con 11.4 ㄷ․

En la serie 1981-2010 (columnas amarillo oscuro) el año más cálido fue 1997 con 13.8 ํC y el más frío 1984 con 12.2 ํ․

Lo más significativo de esta tabla es que, cuando se comparan las series ordenadas, todos y cada uno de los años de la serie 1981-2010 fueron claramente más secos y más cálidos que sus equivalentes en la serie 1951-1980. De entre todos ellos destaca 1989 por ser el más seco y el segundo más cálido de toda la serie 1951-2010.

Utilizando datos diarios, comparado con el periodo 1951-1980, los resultados indican que algunas de las fluctuaciones intra-estacionales del régimen termo-pluviométrico fueron más intensas o incluso nuevas durante el periodo 1981-2010 -dicho esto con ciertas reservas pues la información en esa época era más escasa-, como el segundo verano de final de octubre y el tercer verano de mitad de diciembre, mientras que los mini-veranos de febrero y marzo ya se disfrutaban en el periodo $1951-1980$-ver gráficas del régimen temporal de las págs. 7 y 13-. Si será así en lo sucesivo o si surgirán nuevas fluctuaciones es algo que forma parte intrínseca de la incertidumbre asociada al comportamiento caótico del Clima, tema que se sale de este diagnóstico forense y que es de estudio en el área de las Proyecciones de Cambio Climático.

8.

Al entusiasmo y generosidad de los amigos Colaboradores de la AEMET en Cantabria y alrededores, responsables de la generación de los datos utilizados.

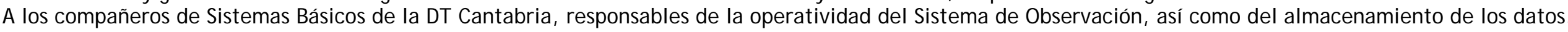
A los compañeros de Climatología de la DT Cantabria, responsables de la calidad y accesibilidad de los datos utilizados.

A todos los compañeros por sus comentarios y sus críticas constructivas. 
9.

Cano R. , 1993

Atlas Climático de la Región Cantábrica

Nota técnica, CMT CAS del Instituto Nacional de Meteorología

Rasilla D. , 1994

Los regímenes de preci pitación en el Norte de la Península Ibérica.

Estudios geográficos. Tomo LV, № 214: 151-181.

Cano R. \& Gutiérrez J . M. , 2004

Relleno de Lagunas y Homogeneización de Series de Precipitación Densas a Escala Diaria

El Clima entre el Mar y la Montaña (ISBN. 84-8102-384-1). AEC, Serie A, Num. 4.

Herrera, S., Frías, M.D., Fernández, J ., Ancell, R., Pons, M. R. \& Gutiérrez, J . M. , 2009

Rejilla de observaciones interpoladas de alta resolución en España para precipitación y temperatura: SpainHR (20km)

CLIVAR. Clima en España: Pasado, presente y futuro | Contribución a un Informe de Evaluación del Cambio Climático Regional 2009

Gutiérrez J.M., 2010

Escenarios Regionales de Cambio Climático en Cantabria: Termopluviometría.

Consej ería de Medio Ambiente. Gobierno de Cantabria

Rodríguez J .J ., 2011

Variabilidad Temporal de Ia Precipitación y la Temperatura en las Comarcas de la Región de Cantabria

Tesis Doctoral, Dep. Geografía de la Universidad de Cantabria

AEMET, 2011

Atlas Climático Ibérico. Temperatura del Aire y Precipitación (1971-2000).

Ministerio de Medio Ambiente y Medio Rural y Marino.

Ancell, R. \& Guillén E. , 2012

Interpolación en áreas de montaña. Aplicación al Parque Nacional de Picos de Europa.

Boletín de la Asociación Meteorológica Española, Enero-2012

Ancell, R. , 2012

Una aproximación al clima del Parque Nacional Picos de Europa

Boletín de la Red de Seguimiento del Cambio Global en Parques Nacionales. Número 2. Otoño-Invierno 2011-2012. 32 páginas. reddeparquesnacionales. mma. es/ parques/ rcg/ documentos/ rcg boletin 02. pdf

Ancell R. \& Guillén E. , 2013

INTERPOLACIÓN GEOESTADISTICA DE TEMPERATURA Y PRECIPITACIÓN A ESCALA DE AGREGACIÓN MENSUAL

Nota Técnica AEMET-Cantabria (Pendiente de publicación) 